Ann. Zootech., I973,I22 (I), II5-II9.

NOTE

\title{
UTILISATION PAR LES VACHES LAITIÈRES DE L'URÉE AJOUTÉE \\ A UNE RATION D'ENSILAGE DE MAÏS RÉCOLTÉ A UN STADE DE MATURITÉ PEU AVANGÉ
}

\author{
M. JOURNET \\ avec la collaboration de B. MArquis \\ Station de Recherches sur l'Élevage des Ruminants, \\ Centre de Recherches de Clermont-Ferrand, I. N.R. A., \\ Saint Genès Champanelle, 63110 Beaumont
}

De nombreux essais ont permis de montrer qu'il était possible de remplacer de 15 à $25 \mathrm{p}$. Ioo de l'azote des tourteaux par de l'azote uréique sans diminuer la production laitière de vaches recevant de l'ensilage de maïs récolté à un stade de maturité assez avancé (plus de 30 p. Ioo de matière sèche) (cf. revues de Coppock et STone, I968; Coppock, I969; Journet et Hoden, 1970). Le présent essai entrepris a pour but d'étudier les possibilités d'utilisation de l'urée, comparativement au tourteau d'arachide, avec un ensilage de mais récolté à 24 p. roo de matière sèche (MS) au stade laiteux du grain, ayant une forte teneur en azote soluble ( 52 p. roo de la MS) et une faible proportion d'épi (33 p. Ioo) (cf. tabl. r).

\section{MATÉRIEL ET MÉTHODES}

Deux aliments complémentaires de la ration d'ensilage de maîs ont été comparés pour satisfaire les besoins azotés des animaux $(60 \mathrm{~g}$ de matières azotées digestibles par $\mathrm{kg}$ de lait à 4 p. Ioo de matières grasses et $0,6 \mathrm{~g}$ de matières azotées digestibles par $\mathrm{kg}$ de poids vif). L'un des aliments était composé de 80 p. Ioo de luzerne déshydratée et 20 p. Ioo d'urée et le deuxième de luzerne déshydratée ( $\mathrm{kg}$ par jour) et de tourteau d'arachide. Les apports azotés du "lot urée " ont été égaux à ceux du "lot tourteau " pour une même production de lait. Les vaches du " lot urée " ont reçu la luzerne déshydratée-urée en 4 distributions par jour pour améliorer l'utilisation de l'urée (la luzerne déshydratée utilisée était d'excellente qualité, is p. Ioo de matières azotées) ; elles ont également reçu un apport supplémentaire d'orge pour équilibrer les apports énergétiques. L'orge broyée a été distribuée en mélange avec le tourteau et la luzerne déshydratée, ou avec la luzerne déshydratée-urée. Les apports énergétiques et azotés ont été ajustés pour chaque vache, chaque semaine, en fonction de la production de lait et des quantités ingérées la semaine précédente.

Deux lots de 9 vaches Frisonnes ( $\mathrm{I}_{4}$ en Ire lactation, 4 en $2^{\mathrm{e}}$ ) ont été appariés à la fin d'une période préexpérimentale de 3 semaines pendant laquelle les animaux recevaient un régime d'ensilage d'herbe et de foin de luzerne. La période expérimentale de I 2 semaines sur l'ensilage 
de maïs distribué ad libitum a été suivie d'une période post-expérimentale de 3 semaines pendant laquelle les vaches ont été mises au pâturage. Le passage du régime pré-expérimental au régime expérimental, puis du régime expérimental au régime post-expérimental s'est effectué au cours de périodes de transition de 2 et I semaines respectivement. Les mesures individuelles suivantes ont été effectuées : quantité de matière sèche ingérée, quantité de lait et taux butyreux journaliers, taux de matières azotées du lait et poids vif hebdomadaire, état d'engraissement au début et à la fin de l'expérience.

\section{TABLEAU I}

Caractéristiques de la plante de maïs et de l'ensilage

\begin{tabular}{|c|c|}
\hline \multicolumn{2}{|l|}{ P. 100 de matière sèche de la plante entière : } \\
\hline - Limbes ......... & 11,6 \\
\hline - Tiges $\ldots \ldots \ldots \ldots \ldots \ldots \ldots$. & 32,8 \\
\hline 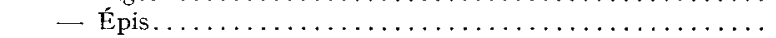 & $33,{ }^{\prime}$ \\
\hline 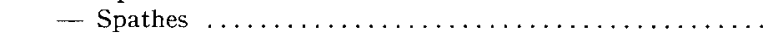 & 19,0 \\
\hline 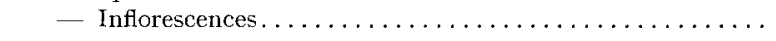 & 5,2 \\
\hline Teneur en matière sèche de l'ensilage $\ldots \ldots \ldots \ldots \ldots \ldots \ldots$ & 24,0 \\
\hline Teneur en matières azotées (p. 100 MS) .. & 9,8 \\
\hline Azote ammoniacal (p. 100 azote total) $\ldots \ldots \ldots \ldots \ldots \ldots$ & 7,9 \\
\hline Azote soluble (p. 100 azote total) ........ & 52,0 \\
\hline \multicolumn{2}{|l|}{ Teneur en acides $(\mathrm{g} / \mathrm{kg}$ MS) : } \\
\hline 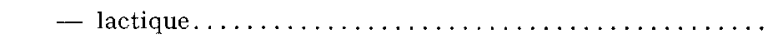 & 53,8 \\
\hline 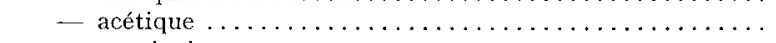 & 52,9 \\
\hline- propionique $\ldots \ldots \ldots \ldots \ldots \ldots \ldots \ldots \ldots \ldots$ & 3,8 \\
\hline 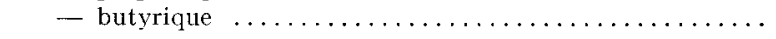 & 2,0 \\
\hline Coefficient de digestibilité de la matière organique $\ldots \ldots \ldots \ldots$ & $72,3\left({ }^{1}\right)$ \\
\hline Coefficient de digestibilité des matières azotées $\ldots \ldots \ldots \ldots \ldots$ & $56,0(\mathbf{1})$ \\
\hline
\end{tabular}

(1) Mesure effectuce sur un lot de ${ }_{t}$ moutons.

\section{RÉSULTATS ET DISCUSSION}

Le mélange luzerne déshydratée-urée et orge broyée a été bien consommé tant que la proportion d'urée dans le mélange ne dépassait pas 6 p. Ioo. La quantité d'urée distribuée a été en moyenne de $182 \mathrm{~g}$ par jour ; elle a varié de $224 \mathrm{~g}$ au début ( 184 à $309 \mathrm{~g}$ selon les vaches) à $137 \mathrm{~g}$ à la fin (92 à $\mathrm{I} 66 \mathrm{~g}$ ). L'azote uréique a représenté $26 \mathrm{p}$. Ioo de l'azote total (30 p. Ioo au début et 23 p. Ioo à la fin de l'expérience).

Par rapport au tourteau, l'urée n'a pas eu d'effet dépressif sur les quantités de maïs ingérées. La production de lait du lot urée a été significativement inférieure $(P<$ o,or $)$ à celle du lot "tourteau " de 2, I kg, soit de I 4 p. Ioo (par analyse de covariance en se rapportant à la période pré-expérimentale). La différence entre les 2 lots s'est surtout " creusée " au cours des 6 premières semaines pendant lesquelles la production de lait a diminué respectivement de 35 et 27 p. roo; elle n'a ensuite diminué que de I 3 et $8 \mathrm{p}$. Ioo pendant les 6 semaines suivantes et cette dernière différence n'a pas été significative. Le taux butyreux a été identique et élevé (près de $4^{\circ} \mathrm{g}$ p. I ooo) dans les 2 lots; le taux de matières azotées du lot tourteau a été supérieur (mais non significativement, par analyse de covariance) de o,8 g p. I ooo à celui du lot urée. Le gain de poids vif des animaux (calculé par régression) au cours de la période expérimentale et l'évolution de l'état d'engraissement des animaux n'ont pas été significativement différents entre les 2 lots (tabl. 2). L'azote du lait a représenté dans chacun des 2 lots 24 et 27 p. 100 de 
l'azote ingéré et $5^{\mathrm{I}}$ p. Ioo de l'azote digestible (après avoir soustrait l'azote digestible nécessaire aux besoins d'entretien). Cette égalité (5I p. IOO) est obtenue lorsqu'on attribue à l'azote de l'urée un coefficient de digestibilité de $77 \mathrm{p}$. Ioo bien qu'il n'ait pas de réelle signification. La faible efficacité apparente d'utilisation de l'azote est en partie due à un apport excédentaire aux besoins qui ont été estimés d'après la quantité de lait 4 p. Ioo sans tenir compte du taux de matières azotées du lait qui a été faible comparativement au taux butyreux. Cette faible efficacité est également la conséquence des normes azotées qui sont élevées, comparativement à celles utilisées dans d'autres pays.

TABIEAU 2

Utilisation comparée des 2 sources azotées : urée et tourteau d'arachide

\begin{tabular}{|c|c|c|c|}
\hline & $\begin{array}{c}\text { Lot } \\
\text { "urcee " }\end{array}$ & $\begin{array}{c}\text { Lot } \\
\text { "tourtean" }\end{array}$ & $\begin{array}{c}\text { Test } \\
\text { de signification }\end{array}$ \\
\hline \multicolumn{4}{|l|}{$\begin{array}{c}\text { Quantités ingérées } \\
\text { (kg de matière sèche par jour) }\end{array}$} \\
\hline Ensilage de maïs $\ldots \ldots \ldots \ldots \ldots \ldots \ldots \ldots \ldots$ & 10,36 & 10,10 & NS \\
\hline 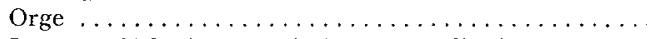 & 1,56 & 1,09 & \\
\hline Luzerne déshydratée-urée ( 20 p. 100 d'urée) . . . . . & 10,91 & & \\
\hline Luzerne déshydratće à 18 p. 100 de mat. azotées . . . . & & 1,00 & \\
\hline Tourteau d'arachide $\ldots \ldots \ldots \ldots \ldots \ldots \ldots \ldots$ & & 1,15 & \\
\hline - Azote total $(N \times 6,25)(g) \ldots \ldots \ldots \ldots \ldots$ & 1790 & 1900 & \\
\hline - Urée à 42 p. 100 d'N $(\mathrm{g}) \ldots \ldots \ldots \ldots \ldots$ & 182 & & \\
\hline - $\mathrm{N}$ uréique p. $100 \mathrm{~N}$ total $\ldots \ldots \ldots \ldots \ldots \ldots$ & 26,0 & & \\
\hline Troductions & & & \\
\hline 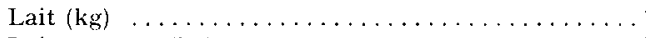 & 13,59 & 15,74 & \\
\hline Lait 4 p. $100(\mathrm{~kg}) \ldots \ldots \ldots \ldots \ldots \ldots \ldots \ldots \ldots \ldots \ldots \ldots$ & $1: 3,33$ & $15,4 \underline{2}$ & $P<0,01$ \\
\hline Natières grasses $(g) \ldots \ldots \ldots \ldots \ldots \ldots \ldots \ldots$ & 552 & 620 & $\mathrm{I}^{>}<0,01$ \\
\hline Taux butyreux $(g$ p. 1000$) \ldots \ldots \ldots \ldots \ldots \ldots \ldots$ & 39,5 & 39,2 & NS \\
\hline Taux de matières azotées $(g$ p. 1000$) \ldots \ldots \ldots$ & 31,3 & 32,6 & NS \\
\hline Poids vif $(\mathrm{kg}) \ldots \ldots \ldots \ldots \ldots \ldots \ldots \ldots \ldots \ldots \ldots \ldots \ldots \ldots \ldots$ & 556 & $5: 9$ & Xs \\
\hline Gain de poids vif $(g / j) \ldots \ldots \ldots \ldots \ldots \ldots$ & $1 \geq 1$ & -11 & NS \\
\hline Variation de l'état d'engraissement (notation de 1 à 5 ) & $\therefore-10,25$ & $+0,31$ & NS \\
\hline
\end{tabular}

Le régime d'ensilage de maïs seul distribué ad libitum et qui constituait le seul fourrage de la ration a été ingéré en quantité modérée : $1,9 \mathrm{~kg}$ de matière sèche par Ioo $\mathrm{kg}$ de poids vif en moyenne dans les 2 lots (de I,66 à 2,36 selon les vaches); la diminution rapide de la production de lait en début d'expérience a été due en partie à la sous-alimentation énergétique, en raison de la surestimation de la valeur de l'ensilage de maïs (cf. ci-après). Cependant, la production de matières grasses a diminué moins rapidement, en raison cle l'accroissement important du taux butyreux de $7 \mathrm{~g}$ p. I ooo en I 2 semaines (fig. I). Malgré la faible persistance de la production laitière, les vaches ont eu tendance à se maintenir à poids constant ou à gagner du poids.

La valeur énergétique de l'ensilage de maïs calculée en tenant compte des besoins énergétiques des animaux $\left(0,38 \mathrm{UF} / \mathrm{kg}\right.$ de lait 4 p. Ioo et $\mathrm{I}, 5+\frac{\mathrm{P}}{200} \mathrm{UF}$ pour l'entretien) et des apports estimés des aliments concentrés complémentaires, a été de $0,72 \mathrm{UF} / \mathrm{kg}$ de MS, alors que la valeur calculéc par la formule de Brefrem (1954), à partir du coefficient rle digestibilité de la matière organique était de $0,79 \mathrm{UF}$. 
L'effet dépressif de l'urée sur la production de lait peut être attribué à la proportion élevée d'azote uréique mais aussi probablement aux caractéristiques de l'ensilage de maïs, en particulier à sa teneur élevée en azote soluble et à sa faible teneur en amidon liées à son stade de
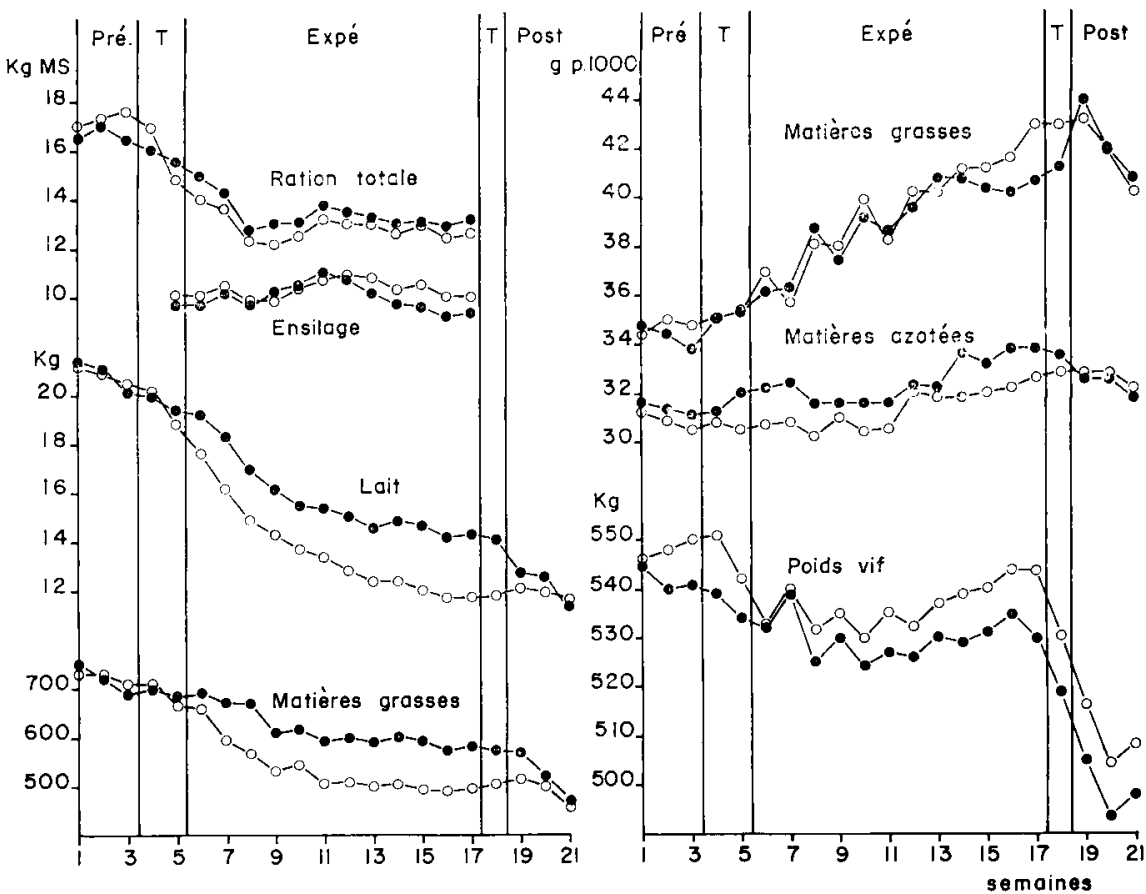

l'IG. I. - Évolution des quantitês ingêrées, de la production de lait et de matières grasse's, des teneurs en matières grasses et en matières azotées du lait et $d u$ poids vif des animaux

$$
\begin{aligned}
& \text { O- urée } \\
& \text { Pré Période pré-expérimentale } \\
& \text { Post } \text { Période post-expérimentale } \\
& \text { Exp. Période expérimentale } \\
& \mathrm{T}
\end{aligned}
$$

maturité peu avancé. En effet, les córéales (orge et maïs) n'ont représenté que $3^{8}$ p. roo de la matière sèche de la ration et la quantité totale d'azote soluble ingérée a été élevée comparativement à la quantité de céréales ( 7 p. roo de la matière sèche des céréales). Ces valeurs sont très inférieures à celles observées dans les expérimentations où l'urée a été bien utilisée (JourNET et al., I970). L'étude de l'aminoacidémic libre du sang a permis de mettre en évidence une diminution de la concentration de certains acides aminés indispensables chez les animaux recevant l'urée comparativement au tourteau, mais ces modifications sont plus faibles que celles observées lors du passage du régime d'ensilage de maïs au pâturage, pendant lequel la concentration en acides aminés indispensables a augmenté de façon importante (CHAMPREDoN et al., I970).

L'effet défavorable de l'urée sur la prołuction de lait, comparativement au tourteau, s'est surtout manifesté en début d'expérience et il doit être attribué à la proportion élevée d'azote uréique à cette période et au temps d'adaptation de la flore microbienne à l'urée. Cet effet a dû être accentué par la diminution plus rapide des quantités d'aliments concentrés distribuées (et donc d'énergie) en début d'expérience dans le lot urée, en raison de la méthode d'ajustement hebdomadaire des quantités d'aliments concentrés offertes en fonction de la production de lait observée. Sur l'ensemble de l'expérience les apports du " lot urée " par rapport au "lot tourteau » ont été inférieurs de 0,70 ITF et I oo $\mathrm{g}$ de matières azctées par jour. 
Cet essai permet d'attirer l'attention sur la nécessité d'utiliser l'urée avec des ensilages de mais à stade de maturité avancé ayant une proportion élevée de grain et une faible proportion d'azote soluble. La proportion d'azote soluble dans la ration devrait probablement être inférieure à $I, 7$ p. Ioo de la matière organique digestible ingérée ou à 3,5 p. roo de la quantité de céréales (y compris le grain contenu dans le maïs ensilé). Cet essai montre également la nécessité de prévoir une période de transition d'au moins i 5 jours pour adapter les animaux à l'urée.

Reçu pour publication en juillet 1972.

\title{
SUMMARY
}

\author{
UTILIZATION BY DAIRY COWS OF UREA ADDED TO A DIE'T \\ OF ENSILED MAIZE HARVESTED AT AN EARLY STAGE OF MATURITY
}

Urea included into dried lucerne at a level of $20 \mathrm{p}$. Ioo was used as a supplement to a diet of maize silage as compared to peanut oil-meal. The milk production of the cows receiving urea was I4 p. Ioo lower. This probably depended on a too high level of urea nitrogen in the diet (25 p. Ioo of total nitrogen), but was especially due to a too low proportion of cereals ( 38 p. roo, maize grain included) because of the insufficient stage of maturity of the maize (24 p. Ioo dry matter, 33 p. Ioo cobs) harvested at an altitude of $800 \mathrm{~m}$.

Urea caused a rapid decrease of the milk production at the beginning of the experiment. This was due in part to a larger reduction of the concentrate supply (and energy supply), determined according to the amount of milk produced. These findings show the role of the time of adaptation of the animals to high urea doses.

\section{RÉFÉRENCES BIBLIOGRAPHIQUES}

Breirem K., 1954. Die Nettoenergic als grundlage der bewertung der futtermittel, in : Nehring K., Ioo jahre Möckern. Die bewertung der futterstoffe und andere probleme der tiernährung. Berlin, Deutsche Akad. der Landwirts chafts wissenchaften, t. II, 97-108.

Champridion C., Pion R., Journet M., ig7o. Influence de la nature des matières azotées alimentaires sur l'amino-acidémie libre des vaches laitières. Ann. Biol. anim. Bioch. Biophys., 10, 5I 7-521.

Coppock C. E., 1969. Problems associated with all corn silage feeding. J. Dairy sci., 52, 848.

Coppock C. E., Stone J. R., r968. Corn silage in the ration of Dairy Cattle. A review. New York state College of Agriculture. Cornell Miscellaneous Bulletin, $\mathbf{8 9}$.

Jounnet M., Hoden A., r97o. Utilisation de l'ensilage de mais pour los vaches laitières. Bull. Tech. Infor, 250, $383-400$. 